$\mathbb{T}$ periodica polytechnica

Chemical Engineering

$54 / 1(2010) 4145$

doi: $10.3311 /$ pp.ch.2010-1.06

web: http://www.pp.bme.hu/ch

(C) Periodica Polytechnica 2010

RESEARCH ARTICLE

\section{New area for distillation: wastewater treatment}

\author{
Katalin Koczka / Péter Mizsey
}

Received 2009-04-11, accepted 2009-06-23

\begin{abstract}
The protection of our environment is an actual and global problem in our time and the wastewater treatment is its important part. It is based on biological treatment. In certain cases, however, due to special regulations, the biological tools are not allowed and other options should be considered and selected. The incineration is an alternative but impact assessment studies show that it can make a serious burden upon the environment as well as it might be an expensive solution. Physico-chemical tools can mean a realistic alternative in such cases. In case of wastewater containing organic solvents (ethanol, ethyl-acetate, toluene, halogenated solvents, etc.), that is typical for wastewaters of medicine and fine chemical industries, the distillation is a powerful tool to remove the volatile polluting compounds. These volatile organic compounds (VOC) can contribute significantly to the chemical oxygen demand (COD) of the wastewater. This contribution we call VOC-COD. The Adsorbable Organically Bound Halogens (AOX) can be removed also with distillation. Industrial case studies show that the distillation offers a realistic alternative and the recovered solvents can be also utilised. The $C O D$ can be reduced in certain cases below the emission limit (1000 mg/lit) and the AOX, after the physico-chemical treatment, is less than $8 \mathrm{mg} / \mathrm{lit}$.
\end{abstract}

\section{Keywords}

wastewater treatment $\cdot$ distillation $\cdot$ COD reduction $\cdot$ industrial case study

\section{Katalin Koczka}

Department of Chemical and Environmental Process Engineering, BME, H1111, Budapest, Budafoki út 8, Hungary

e-mail:kkoczka@mail.bme.hu

\section{Péter Mizsey}

Department of Chemical and Environmental Process Engineering, BME, H1111, Budapest, Budafoki út 8, Hungary

\section{Introduction}

In recent years, in every area of the chemical industry the environmental protection has become a more and more severe activity of the industrial production chain. The most significant problem of the environmental protection is the waste and the treatment of waste. There might be several solutions for the waste problem:

1 waste minimisation, possibly elimination in the frame of a so called "zero emission technology" is the most preferred solution but sometimes it is inevitable that waste arises;

2 if waste is arisen it should be maintained that this waste finds application somewhere in another technology. To solve this problem, one should work on multiple levels, that is, in a factory there might be several plants, and the waste of one plant can be, preferably, utilised in another plant [1];

3 if we cannot find application for a waste, we should try to treat it so that the emission is minimised. That means, the end-of-pipe treatment methods should be also developed.

It is the task of the recent engineer community to elaborate how the already existing industrial processes can be reconstructed, replaced or substituted that they fulfil the strict environmental regulations. On the long term, the final objective of environmental protection is the invention of "zero emission technologies".

Wastewaters or process waters belong to the category where the wastes cannot be eliminated and effective end-of-pipe methods should be considered instead of direct emission [1]. At the application of the end-of-pipe methods special attention is to be paid to the possible recycling of the recovered contaminating compounds, if possible.

In the case of wastewaters the biological treatment with combination of mechanical processes is the traditionally favoured option. The biological treatment alternatives, however, cannot be always applied because of their inherent limitations [2]-[5]. On the other hand, local governments do not allow their applications in strongly populated areas because the required distance to protect the human population is usually missing. Therefore, in chemical plants, located in populated areas, other al- 
ternatives should be considered, like incineration or physicochemical tools. Since the incineration excludes the option of recovery it is the least favoured option.

Important physico-chemical tools are the different separation units that are based on the different relative volatilities of the compounds $[6]-[8]$. If the contamination is more volatile than the water or forms heteroazeotropes with it, the stripping and the different kinds of distillation can be successfully applied. These physico-chemical tools have the advantage that contaminating volatile organic compounds (VOC) can be also recovered and reused [9]-[12]. If non-volatile compounds are also present, the problem should be solved with other tools e.g. membrane processes or wet oxidation [13]-[16]. All this is an increasing challenge for engineers to develop new processes which enables factories to fulfil the environmental regulations [17]. In different industries there are such wastewaters which contain organic solvents and chlorinated hydrocarbons. These compounds raise both the Chemical Oxygen Demand (COD) and Adsorbable Organically Bound Halogens (AOX) value.

The different areas of the application of physico-chemical tools can be summarised:

- nitrogen content: desorption with gas or steam,

- VOC: stripping, rectification if the contamination is going to be recovered in concentrated form for possible reuse

- $A O X$ : stripping with steam or air, desorption

- heavy metals: membrane filtration [18]

- high salt content: evaporation, to minimise the quantity to be incinerated.

The treatment of waters with high salt content can be treated in several other ways, e.g. wet oxidation.

\section{The distillation in the wastewater treatment}

The distillation has already found many application areas of separation and its ability can be well utilised also for wastewater treatment. The separation of the VOC decreases also the COD and in several industrial cases this COD reduction is usually satisfactory (COD reduction due to the separation of the VOC can be called VOC-COD). Other important task of the wastewater treatment is the separation of adsorbable organically bound halogens (AOX). This separation task can be also completed with distillation but careful processing should be followed. This paper focuses on different fugacity based methods.

Industrial wastewaters are usually multi component mixtures, thus their treatment with distillation and/or other physicochemical tools might pose some difficulties. They usually contain alcohols (methanol, ethanol, isopropanol, benzyl alcohol), organic solvents (acetone, ethyl acetate), chlorinated hydrocarbons (dichloromethane, benzyl chloride) and aromatic hydrocarbons.

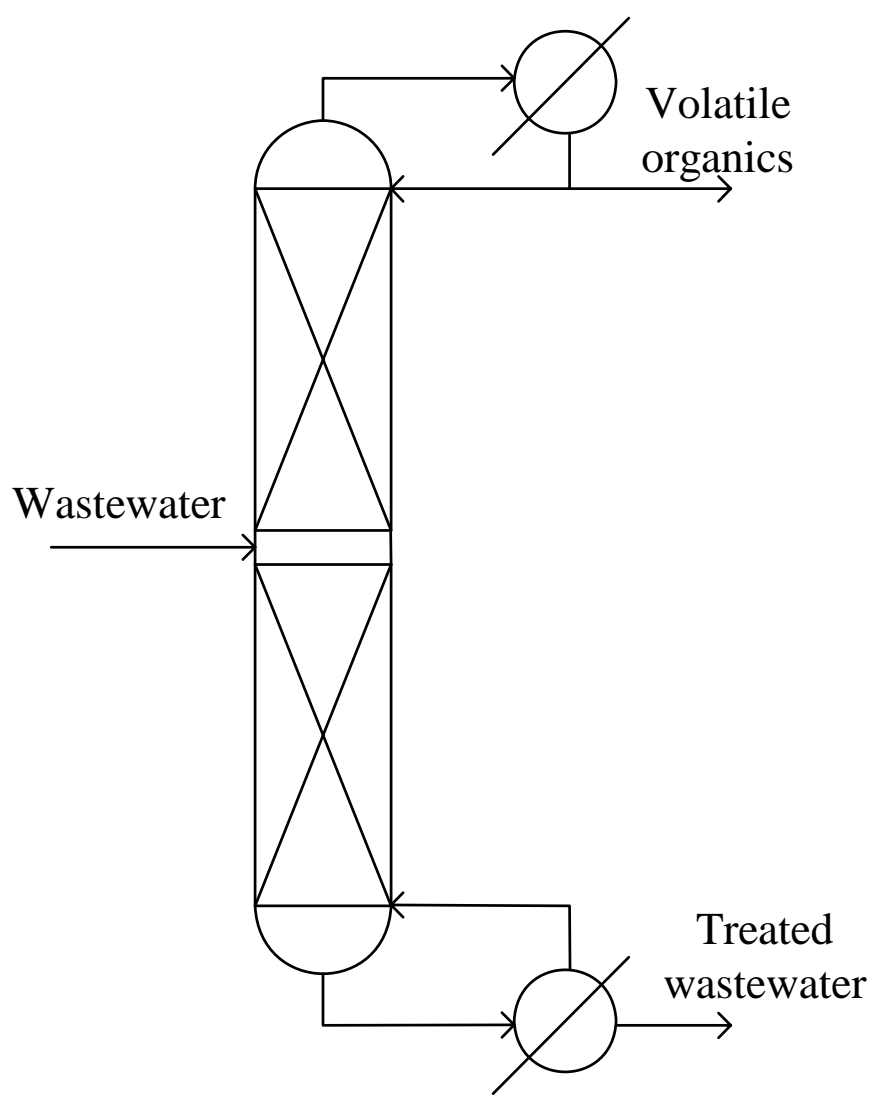

Fig. 1. Schematic distillation column

If distillation is applied, this should fulfil two requirements: (i) the bottom product, the cleaned wastewater, should be VOC and/or AOX free or below the limits, (ii) the top product, distillate, should be as concentrated as possible so that the VOC could be used for other purposes or, if it is a waste, the minimal amount should be incinerated. Considering these two requirements, the rectification offers a powerful solution (Fig. 1).

\section{Experiments}

In our work, several industrial wastewaters of medicine and fine chemical industries are investigated, analyzed, and classified according to different aspects. The tasks are the VOC separation and the VOC-COD and the AOX reduction. Typical mixtures are selected as representatives. Table 1 shows their main feed compositions and initial COD values measured. The VOCCOD can be calculated on the basis of the feed composition with the help of the ChemCAD flowsheeting software package [19]. This calculations show that the VOC-COD is close to the measured COD values, that indicates good chances for a wastewater treatment with rectification. These wastewaters usually contain other water-soluble materials. It can happen, that the bottom product does not fulfil the release limit of COD, in spite that it is solvents free. This can be due to the water-soluble heavy compounds. The remove of water-soluble materials is another problem which can be solved by nanofiltration or reverse osmosis. The wet oxidation can be also considered.

After the detailed analysis of the wastewaters selected, sim- 
Tab. 1. Feed composition of mixtures selected

\begin{tabular}{lcccccc}
\hline Major organic solvents wt\% & Mixture1 & Mixture2 & Mixture3 & Mixture4 & Mixture5 & Mixture6 \\
\hline Methanol (MeOH) & & 1.9 & 0.06 & & 0.01 & 0.40 \\
Ethanol (EtOH) & 0.02 & 2.8 & 0.15 & 0.98 & 1.33 & 0.26 \\
Isopropanol (IPA) & 7.85 & 8.7 & 17.40 & & & \\
Dichloromethane (DKM) & & & & 0.09 & 0.52 & 0.34 \\
Ethyl acetate(EtAc) & 0.08 & & & 0.30 & 1.95 & 0.44 \\
Benzyl alcohol (BA) & & & & & 0.25 & 0.11 \\
Benzene (Be) & 159140 & 167860 & 546450 & 15515 & 23100 & 38470 \\
COD [mg/L] & & & & & & \\
\hline
\end{tabular}

ple laboratory distillation experiments are carried out. For the VOC analytics a gas chromatograph equipped with a flame ionization detector is used. The distillate water content is measured by Karl-Fischer titration. The COD is measured by ISO 6060:1991and/or AOX value is also determined.

Laboratory rectification is also completed. On the basis of these data the major parameters of a rectification column can be determined. These parameters are the number of theoretical plates, feed tray location, reflux ratio, distillate - feed ratio. Considering the features of the separation problem, structured packing is selected as column internal. This has the advantage that a scale up is easy to realise or, with other words, the separation efficiency of a pilot plant equipment and an industrial one can be directly compared if both are filled in with the same structured packing.

As a scale-up, a pilot plant column is built and the separation is tested in this scale as well before industrial design. The separation tasks are the removal of VOC and the realisation of the AOX release limit.

According to the experiments a pilot plant distillation column with 15 theoretical plates is suitable for the wastewater treatment option. The feed tray location is the middle of column.

\section{Results and discussion}

Industrial mixtures are analyzed and classified according to different aspects. These aspects are associated with the polluting compounds. The most dangerous and followed pollutions are organically bound halogens. In our work we can differentiate the wastewaters if they contain halogens or not. This is the major fact considered from the point of view of environmental regulations and the factory with high halogen emission (AOX) can be fined and/or closed.

The COD and the VOC emissions are also followed and fined and their emission must be also avoided. Therefore, in our research work we also follow this classification and we differentiate the wastewaters whether they contain halogens or not.

\subsection{Wasteweaters with no halogen content}

The typical feature of Mixture 1-3 are the relatively high alcohol content. Two of them form homogeneous azeotropes $(\mathrm{EtOH}$, IPA), and EtAc form a heterogeneus azeotrop with the water, so a water free distillate is impossible with an ordinary rectification. After laboratory experiments and distillation, these wastewaters are also treated in the rectification column of pilot plant size.

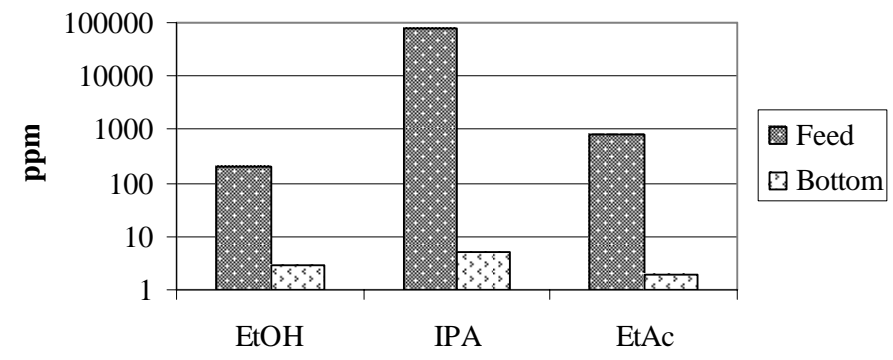

Fig. 2. Feed and bottom compositions of Mixture 1

Fig. 2 shows the efficiency of the separation realised in the pilot plant scale rectification column. (Please, pay attention to the logarithmic scale of the presentations.) The distillate/feed ratio is relativly low, it is $9 \%$. It can be seen, that the composition of the main organic solvents are less then $10 \mathrm{ppm}$. The COD of the treated wastewater is reduced from $159,140 \mathrm{mg} / \mathrm{lit}$ to $950 \mathrm{mg} / \mathrm{lit}$. It can be said, that the bottom product is almost volatile organic compounds free, and the COD value meets the release requirements.

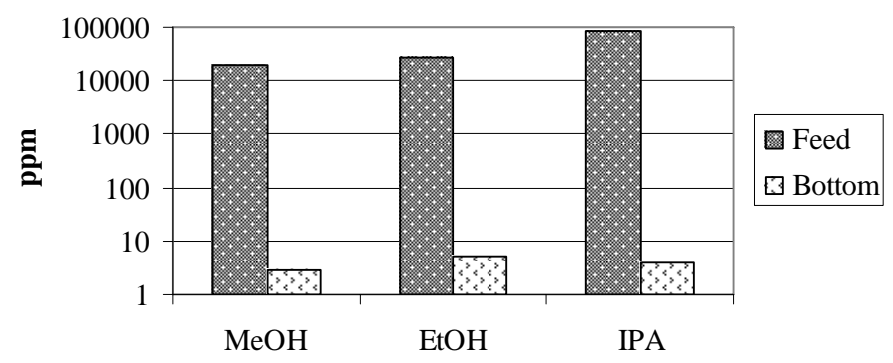

Fig. 3. Feed and bottom compositions of Mixture 2

In the case of Mixture 2, the distillate/feed ratio is relatively high, it is $16 \%$, but it is necessary for azeotropes, and the reflux ratio is 20. Fig. 3 shows the efficiency of the rectification. It can be seen that the remaining alcohol content is less then $10 \mathrm{ppm}$ for all the three alcohols. The COD of the treated wastewater is reduced from $167,860 \mathrm{mg} / \mathrm{lit}$ to $3,500 \mathrm{mg} / \mathrm{lit}$.

Fig. 4 shows the composition of Mixture 3. The distillate/feed ratio is relatively high, it is $26 \%$, but it is necessary 


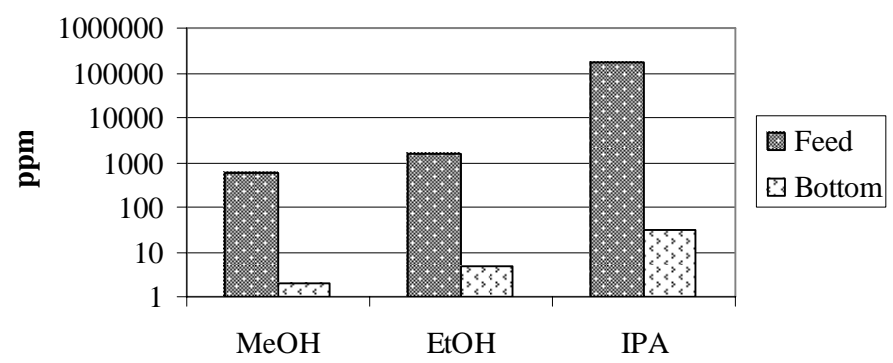

Fig. 4. Feed and bottom compositions of Mixture 3

for azeotropes, and the reflux ratio is 20 . The remining IPA content is a higher than $10 \mathrm{ppm}$ but the top procut has $30 \mathrm{wt} \%$ water content, thus it is not reasonable to increase the distillate/feed ratio. The COD of this mixture is reduced from $546,450 \mathrm{mg} / \mathrm{lit}$ to $17,000 \mathrm{mg} / \mathrm{lit}$. This result is due to the non volatile compounds being in the wastewater. Here further treatment is necessary, other physico-chemical tools can be applied like wet oxidation, membrane filtration etc.

\subsection{Wasteweaters with halogen content}

Wastewaters with halogen content are more difficult to be treated. In the cases investigated (Table 11. Mixtures 4-6), the halogen content is in the form of dichloromethane (DKM). Every mixture contains DKM and EtAc, and both have limited solubility in the water. In the case of this three mixtures, homogeneous and/or heterogeneous azeotropes make the seaparaition even more difficult.

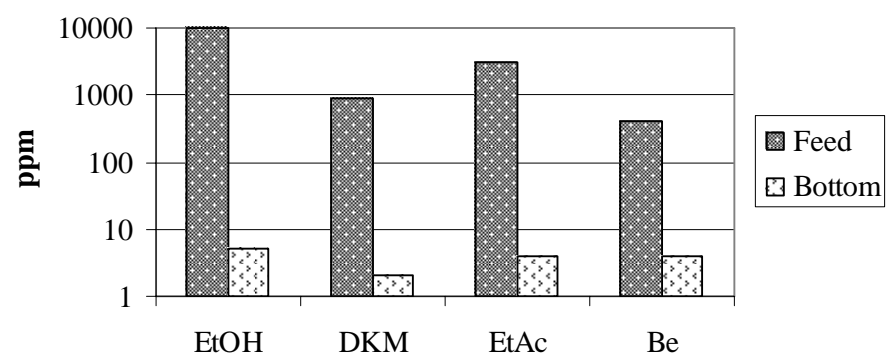

Fig. 5. Feed and bottom composition of Mixture 4

Fig. 5 shows the efficiency of the separation realised in the pilot plant scale rectification column. Mixture 4 contains both homogeneous and heterogeneous azeotropes. The distillate/feed ratio is relatively low, it is $1,6 \%$, but it is sufficient to eliminate the volatile organic solvents from the wastewater. The reflux ratio is 20. The results show that the AOX limit, that is, the DKM concentration can be decreased below the release limit, $8 \mathrm{ppm}$. The COD is reduced from $15,515 \mathrm{mg} /$ lit to $990 \mathrm{mg} / \mathrm{lit}$. It can be said, that the bottom product is practically volatile organic compounds free, and the COD value meets the requirements.

Fig. 6 shows the results of the separation. The distillate/feed ratio is about $10 \%$, the reflux ratio is between $10-20$. The results show that the AOX limit, that is, the DKM concentration can be decreased below $5 \mathrm{ppm}$. The COD is reduced from 23,100 $\mathrm{mg} / \mathrm{lit}$ to $2,600 \mathrm{mg} / \mathrm{lit}$. The remaining COD can be due to the non-volatile compounds.

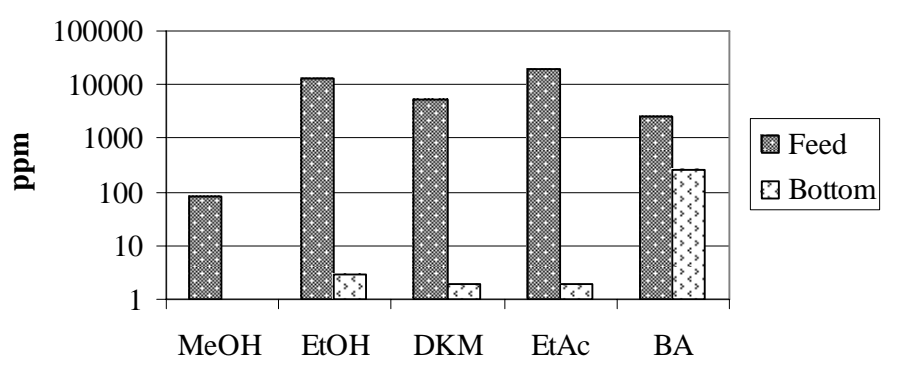

Fig. 6. Feed and bottom composition of Mixture 5

The benzyl-alcohol (BA) concentration could be further reduced, if needed. Considering that the benzyl-alcohol forms a heterogeneous azeotrop with the water $(7.8 \mathrm{wt} \%$ BA, $92.2 \mathrm{wt} \%$ water), if it is also removed from the bottom product (cleaned wastewater) it would be associated with significantly more water in the distillate. Considering that some organic compounds form heterogeneous mixtures with the water, similar to BA, a heterogeneous azeotropic distillation could help in this issue. On the contrary, considering also that other organic compounds form homogeneous mixtures with the water, the heterogeneous azeotropic distillation couldn't help in those cases. Therefore a trade-off must be set up, either the BA remains in the wastewater and other physico-chemical tools should be applied or two subsequent distillations (common and heterogeneous azeotropic ones) should be applied.

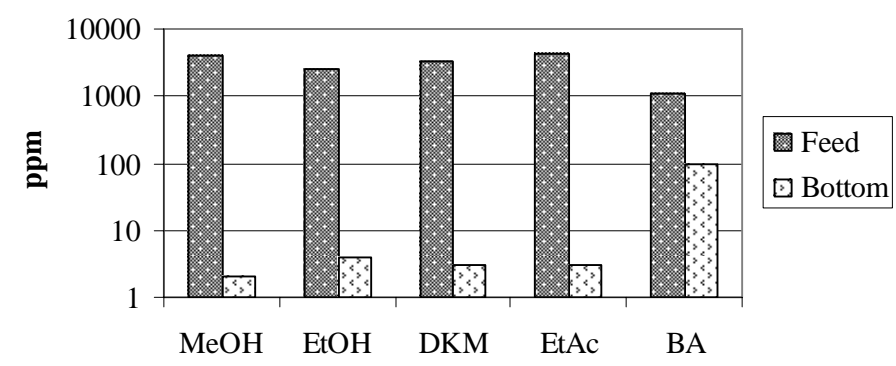

Fig. 7. Feed and bottom composition of Mixture 6

Mixture 6 is similar to Mixture 5, because the organic solvent contents are the same. The result of rectification can be seen in Fig. 7. The distillate/feed ratio is $6 \%$, and reflux ratio is 20 . The results show that the AOX limit, that is, the DKM concentration can be decreased below the release limit. The COD is reduced from $38,470 \mathrm{mg} / \mathrm{lit}$ to $10,670 \mathrm{mg} / \mathrm{lit}$. The remaining COD can be due to the non-volatile compounds. The benzyl-alcohol concentration could be further reduced, but this will not solve the required $\mathrm{COD}$ value reduction for sure. Here further reduction is necessary, other physico-chemical tools can be applied like wet oxidation, membrane filtration etc.

According to our industrial practice, the BA remaining in the wastewater does not disturb significantly the emissions since the different wastewaters are mixed and they can balance each other features.

Fig. 8 shows the COD value reduction with distillation for the wastewaters investigated. Mixtures 1 and 4 give good COD value after rectification, less than $1000 \mathrm{mg} / \mathrm{lit}$, and Mixtures 2 


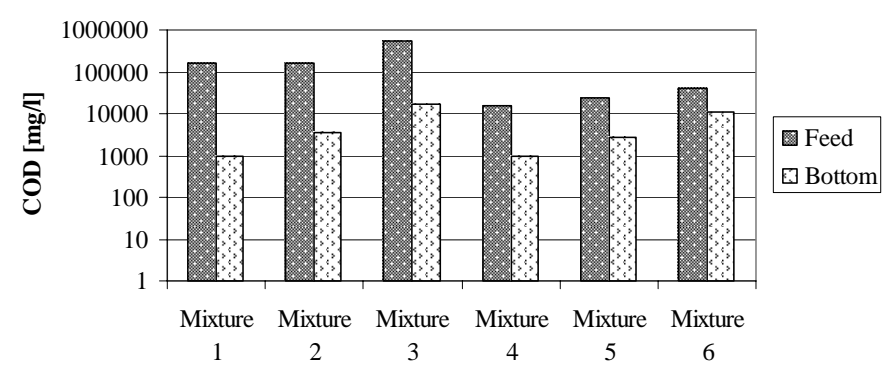

Fig. 8. Feed and bottom COD value of Mixtures

and 5 are fairly close to the required COD value. Mixtures 3 and 6 do not meet the requirement.

It can be said, that the volatile organic compounds do not present really high difficulties for the rectification. However, if there are the non-volatile compounds in the wastewater they cannot be usually eliminated with rectification, unless they form minimum boiling azeotropes with water like benzyl-alcohol. If such compounds should be removed from the wastewater other physico-chemical tools can be also considered like water oxidation or membrane filtration techniques.

\section{Conclusions}

Industrial case studies show that wastewaters with significant VOC pollution and organically bound halogen content (AOX) can be successfully treated with rectification. The VOC-COD can be practically eliminated, that is, the composition of VOCs is less then $10 \mathrm{ppm}$. Heavier polluting organic compounds can be also removed if they form heterogeneous azeotropes with water, since such azeotropes are always minimal ones. The halogen content (AOX) can be reduced below the emission limit of $8 \mathrm{ppm}$.

\section{References}

1 Mizsey $\mathbf{P}$, Waste reduction in the chemical industry - a two level problem, $\mathbf{J}$ Hazard Mater 37 (1994), 1-13.

2 Getzner M, The quantitative and qualitative impacts of clean technologies on employment, J Clean Prod 10 (2002), 305-319.

3 Belis-Bergouignan MC, Oltra V, Jean MS, Trajektories towards clean technology: example of volatile organic compound emission reductions, Ecol Econ 48 (2004), 201-220.

4 Lapara TM, Nakatsu CH, Pantea LM, Alleman JE, Aerobic biological treatment of a pharmaceutical wastewater: effect of temperature on COD removal and bacterial community development, Wat. Res. 35 (2001), no. 18, 4417-4425.

5 Lin SH, Kiang CD, Combined physical, chemical and biological treatments of wastewater containing organics form a semiconductor plant, J Hazard Mater B97 (2003), 159-171.

6 McCabe WL, Smith JC, Unit operation of chemical engineering (1967), 540-609. $2^{\text {nd }}$ ed.

7 Newman SA, Thermodynamics of Aqueous Systems with Industrial Applications, ACS Symposium series 133, 1980, pp. 15-49.

8 Strigle RFJr., Random Packings and Packed Towers, Design and Applications, Gulf Publishing Company, Houston, 1987.

9 Dursun D, Sengul F, Waste minimization study in a solvent-based paint manufacturing plant, Resour Conserv Recy 47 (2006), 316-331.
10 Lucia A, Finger V, Co-solvent selection and recovery, Adv Environ Res 8 (2003), 197-211.

11 Masango $\mathbf{P}$, Cleaner production of essential oils by steam distillation, $\mathbf{J}$ Clean Prod 13 (2005), 833-839.

12 Pinto R. T. P., Lintomen L, Luz Jr L.F.L, Wolf-Maciel M. R, Strategies for recovering phenol from wastewater: thermodynamic evaluation and environmental concerns, Fluid Phase Equilibr 228-229 (2005), 447-457.

13 Bes-Piá A, Iborra-Clar MI, Iborra-Clar A, Mendoza-Roca J A, CuartasUribe B, Alcaina-Miranda MI, Nanofiltration of textile industra wastewater using a physicochemical process as a pre-treatment, Desalination 178 (2005), 343-349.

14 Levec J, Pintar A, Catalytic wet-air oxidation processes: A review, Catal Today 124 (2007), 172-184.

15 Sangave PC, Gogate PR, Pandit AB, Combination of ozonization with conventional aerobic oxidation for distillery wastewater treatment, Chemosphere 68 (2007), 34-41.

16 Suarez-Odeja ME, Stüber F, Fortuny A, Fabregat A, Carrera J, Font $\mathbf{J}$, Catalytic wet air oxidation of substituted phenols using activated carbon as catalyst, Appl Catal B-Environ 58 (2005), 105-114.

$17 \mathbf{H e} \mathbf{C H}$, Gao YH, Yang SH, Edwards DW, Optimization of the process for recovering caprolactam from wastewater in a pulsed-sieve-plate column using green design methodologies, J Loss Prevent Proc 17 (2004), 195-204.

18 Pauer V, Cséfalvay E, Mizsey $\mathbf{P}$, Removal of copper from process waters by reverse osmosis, Technical Chemistry Days (2007), 203-206. (In Hungarian).

19 available at www. chemcad.net 